\title{
Advanced Techniques for Networked Systems with Applications
}

\author{
Zhiyong Chen, ${ }^{1}$ Haitao Zhang, ${ }^{2}$ Lijun Zhu, ${ }^{3}$ Steffi Knorn, ${ }^{4}$ and Zhao Cheng ${ }^{5}$ \\ ${ }^{1}$ School of Electrical Engineering and Computer Science, University of Newcastle, Callaghan, NSW, Australia \\ ${ }^{2}$ Department of Control Science and Engineering, Huazhong University of Science and Technology, Wuhan, China \\ ${ }^{3}$ Department of Electrical and Electronic Engineering, University of Hong Kong, Pokfulam, Hong Kong \\ ${ }^{4}$ Department of Engineering Sciences, Uppsala University, Uppsala, Sweden \\ ${ }^{5}$ Thayer School of Engineering, Dartmouth College, Hanover, NH, USA \\ Correspondence should be addressed to Zhiyong Chen; zhiyong.chen@newcastle.edu.au
}

Received 13 December 2016; Accepted 15 December 2016; Published 12 April 2017

Copyright (C) 2017 Zhiyong Chen et al. This is an open access article distributed under the Creative Commons Attribution License, which permits unrestricted use, distribution, and reproduction in any medium, provided the original work is properly cited.

Cooperative control of networked systems is a fast-growing field, in which mathematical methods are typically used to investigate the theories and algorithms that address efficiency, availability, and robustness of networked systems. Main aspects of rich collaborative behaviors of networked systems include agent dynamics, network communication, and interagent dynamic coupling.

The objective of this special issue is for scientists, engineers, and practitioners to present their latest theoretical and technological achievements on networked systems. It aims at identifying sound theoretical foundation and promising technological solutions to analysis, control, and applications of networked systems.

This special issue has received many submissions. The authors of the papers submitted to this special issue are from Canada, China, Iran, Korean, United Kingdom, United State, Vietnam, and so on. After rigorous peer-review process, six papers have been accepted for publication. These papers present recent research processes in multirobot cooperation control, fault detection, and network communication. The acceptance rate of this special issue is $13 \%$.

In the field of multirobot cooperative control, C. Ruan et al. develop a method to form robust synchronization in the networked system of unmanned combat aerials vehicles (UCAVs). The route temporary blind avoidance (RTBA) model is proposed to prevent the combat aerials vehicles from physical and functional bind. The synchronization is achieved through maximizing the efficiency of UCAV formation in terms of connectivity and delay robustness.

C. Peng et al. investigate the attitude motion synchronization problem for multiple robotic helicopters with three degrees of freedom. The directed graph is used to model the communication topology for the multirobot system. Stability and tracking performance of the whole network is improved by estimating the expected control term and the synchronization error in each individual robot with a finite-time convergent estimator.

Chaotic time series prediction is a challenging problem for neural networks due to its uncertainty. Q. Li and R.-C. Lin Chaotic propose the self-constructing fuzzy neural network (SCFNN) for time series prediction. This SCFNN extends from the fuzzy neural network by conducting the structure learning and parameter learning concurrently and can achieve similar performance with less number of hidden layer nodes.

In order to provide quality-adaptive mobile video streaming services in MIMO-capable heterogeneous wireless access networks, H. Oh extends from the FMIPv6 method whose performance is affected due to large prediction inaccuracy in the case of sudden direction change of the mobile nodes. By optimizing video quality and handover delayer with more appropriate selected metrics, the proposed method is able to provide quality-adaptive service for handing over and forwarding mobile video streaming in MIMO-capable heterogeneous wireless access networks.

X. Ren and M. He developed the Appropriate Degree Gossip Detection (ADGD) which discovers faulty links and peers through depth-first search on the peer degree. In addition, a routing failure restoration strategy (RFRS) that handles single-link failure, multilink failure, and peer failure is proposed to improve the reliability of the P2P dynamic systems.

In addition, N. H. A. Nguyen et al. address stability analysis and control synthesis for the semi-Markovian Jump 
systems (S-MJSs) with the more applicable feature, uncertain probability intensity. The boundary constraints of the probability intensity are discussed and they are related to the basis of time-varying transition rates. The authors also investigate the effectiveness of the control design of the S-MJSs.

\section{Acknowledgments}

We appreciate the authors for their contributions to this special issue. Special thanks to the reviewers for their valuable feedback, which helps improve the quality of the papers submitted.

Zhiyong Chen Haitao Zhang

Lijun Zhu Steffi Knorn Zhao Cheng 


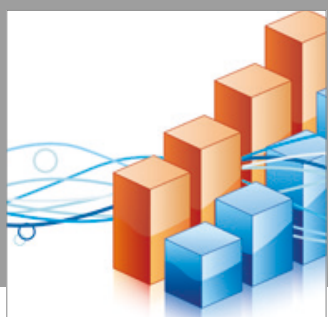

Advances in

Operations Research

vatersals

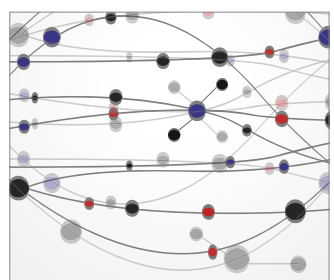

\section{The Scientific} World Journal
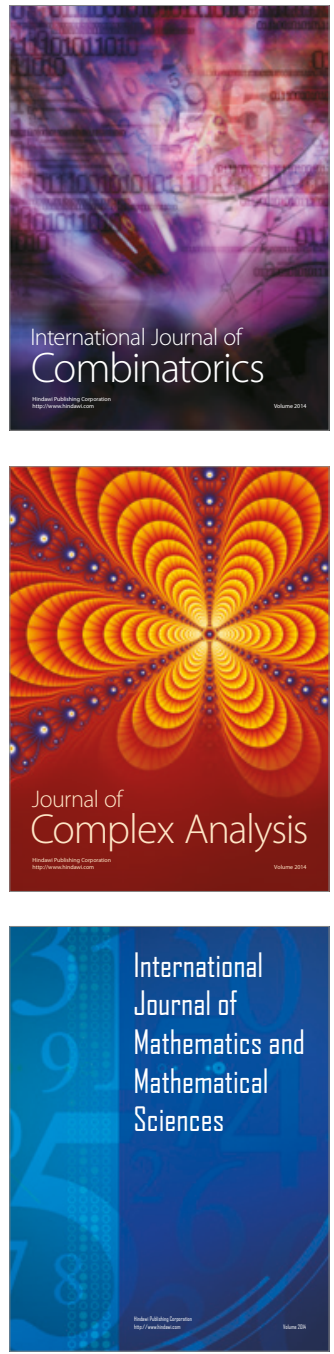
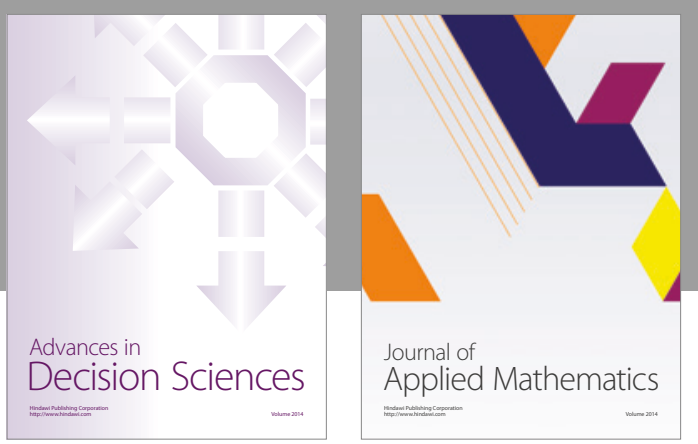

Algebra

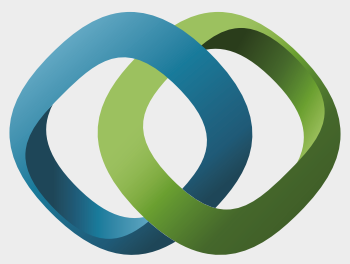

\section{Hindawi}

Submit your manuscripts at

https://www.hindawi.com
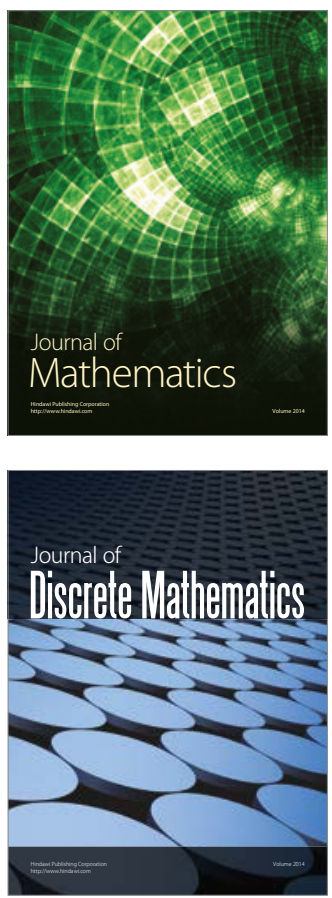

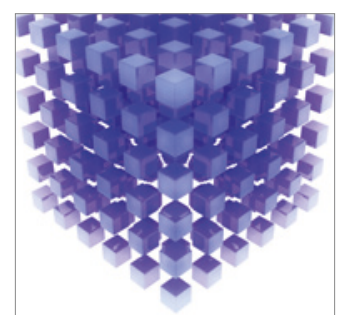

Mathematical Problems in Engineering
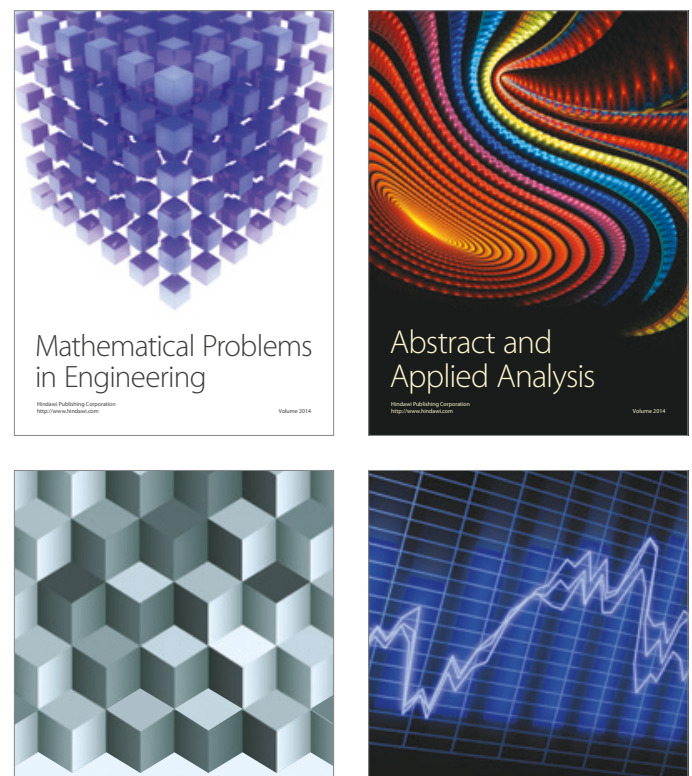

Journal of

Function Spaces

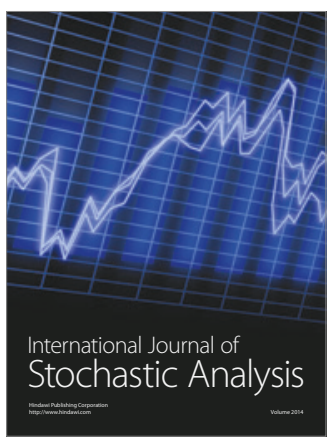

Probability and Statistics
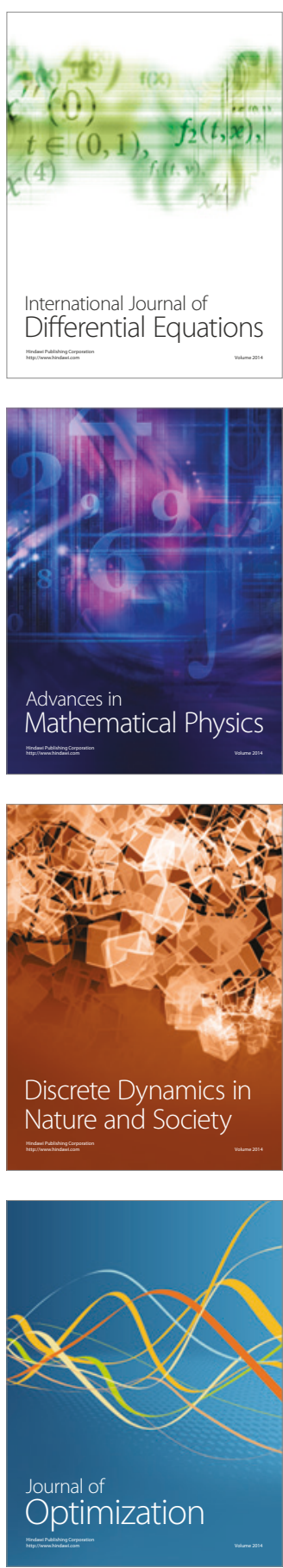